\title{
Interpolation Algorithm and Mathematical Model in Automated Welding of Saddle-Shaped Weld
}

\author{
Lianghao Xue (iD, Min Wei $(\mathbb{D}$, Tao Yang, Yongxin Lu, Ningqiang Shi, and Zhiyang Zhang \\ Mechanical and Electrical Engineering College, Shihezi University, Shihezi 832000, China \\ Correspondence should be addressed to Min Wei; 1448237074@qq.com
}

Received 25 October 2017; Accepted 14 January 2018; Published 1 March 2018

Academic Editor: Ricardo Perera

Copyright (C) 2018 Lianghao Xue et al. This is an open access article distributed under the Creative Commons Attribution License, which permits unrestricted use, distribution, and reproduction in any medium, provided the original work is properly cited.

\begin{abstract}
This paper presents welding torch pose model and interpolation algorithm of trajectory control of saddle-shaped weld formed by intersection of two pipes; the working principle, interpolation algorithm, welding experiment, and simulation result of the automatic welding system of the saddle-shaped weld are described. A variable angle interpolation method is used to control the trajectory and pose of the welding torch, which guarantees the constant linear terminal velocity. The mathematical model of the trajectory and pose of welding torch are established. Simulation and experiment have been carried out to verify the effectiveness of the proposed algorithm and mathematical model. The results demonstrate that the interpolation algorithm is well within the interpolation requirements of the saddle-shaped weld and ideal feed rate stability.
\end{abstract}

\section{Introduction}

In the production of pressure vessels, the welding problem between the cylinder and the pipe is often encountered, and sometimes a pressure vessel needs to weld dozens or even hundreds of pipes. In the project, the intersecting line, called the three-dimensional standard saddle-shaped weld, is formed by the combination of two orthogonal cylinders. Also, according to the different groove form, the multilayer continuous welding is required $[1,2]$. Saddle-shaped weld is a complex space curve. In order to complete the welding work of the saddle seam, manual welding operation is still widely adopted. However, by doing this the welding quality and efficiency requirements are uncertain. Thus, the automation of the welding process is greatly significant.

With the development of automatic welding system, obvious progress has been made in recent years. According to the welding process of saddle seam, there are two ways to build the automated welding system. One is to equip welding robots in their welding system; the other is to develop specially designed automated welding system. One of the most promising ways is to adopt proper curve interpolation algorithm in automated welding system [3]. Huo et al. [4] presented a real-time interpolation algorithm, which fits the intersecting curve with step-controllable segments. Ren et al. [5] established a geometrical model of the interpolation pipe's weld seam and integrated the posture of the welding torch. Zhiming et al. [6] presented a real-time interpolation algorithm for NURBS curves; the proposed interpolator can maintain small contour errors and feed rate fluctuations. Real-time nonuniform rational B-spline interpolation algorithms were also introduced [7-9]. Tam et al. [10] proposed an algorithm for the interpolation of hybrid curves, based on interpolation of the projection of the hybrid curve into the parametric domain. Srijuntongsiri [11] proposed a new iterative hybrid algorithm to detect all intersections in the domain for nondegenerate and non-ill-posed cases. Direct interpolation algorithms $[12,13]$ do not have satisfied linear velocity control ability because welding quality is very sensitive. Only interpolation algorithm with ideal trajectory precision and linear velocity control ability can meet requirements of welding.

In this paper, a spatial interpolation algorithm for the saddle-shaped curve is proposed by using the variable angle interpolation control method for the interpolation stepping angle and establishes welding torch pose model and trajectory model based on space analytic geometry. Simulation of the interpolation algorithm has been carried out to verify the 


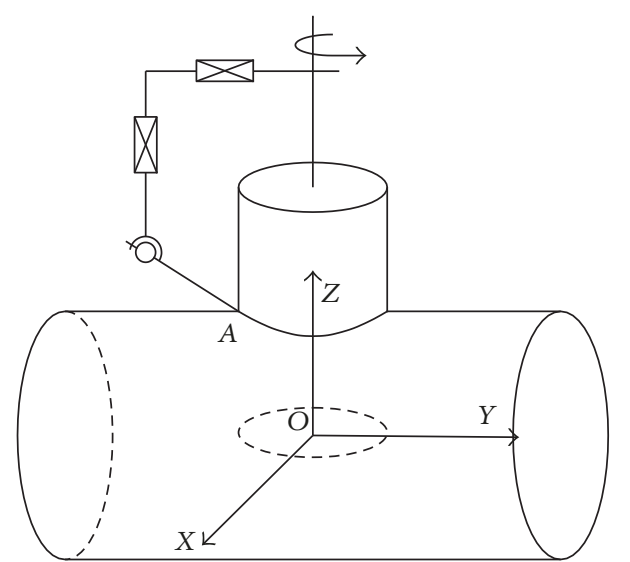

FIGURE 1: Saddle weld and the reference frame configuration.

correctness of the proposed algorithm and mathematical model. It demonstrates that algorithm has high trajectory precision and the mathematical model meets the requirements of welding.

\section{Working Principle of Welding Machine}

In this paper, the typical saddle-shaped weld is formed by the combination of two orthogonal cylinders and the pipe is taken as an example to illustrate the working principle of the saddle-shaped welding system, as shown in Figure 1. The coordinate system $O-x y z$ is established by the intersection of the cylinder and the axis of the pipe, selecting the saddleshaped weld left side of the highest point $A$ as the starting point of welding. The $x$ - and $y$-axis two directions are driven by the entire welding system to achieve rotary motion. The $z$-axis direction is driven by the torch to achieve up and down reciprocating motion, which is also as a compensation movement to achieve saddle drop compensation. At the same time, the upper and lower movement of the output end are installed with angle adjustment mechanism; the welding torch is adjusted in real time with the rotary motion.

According to the characteristic that this kind of weld joint in the horizontal $x \mathrm{O} y$ surface is round, based on the twodimensional plane circular interpolation algorithm, the interpolation of saddle space weld can be decomposed into two two-dimensional interpolations [4]. The first interpolation is the $z$-axis vertical direction interpolation, and the second interpolation is an interpolation of horizontal rotation angel $[14,15]$.

\section{Research of Saddle-Shaped Weld Interpolation Algorithm}

Given that the radius of the cylinder is $R$ and the radius of the pipe is $r$, the projection of the saddle-shaped weld in the $x O y$ plane is shown as in Figure 2, $\theta$ being the angle parameter that can uniquely determine the pose of the point on the saddleshaped space curve.

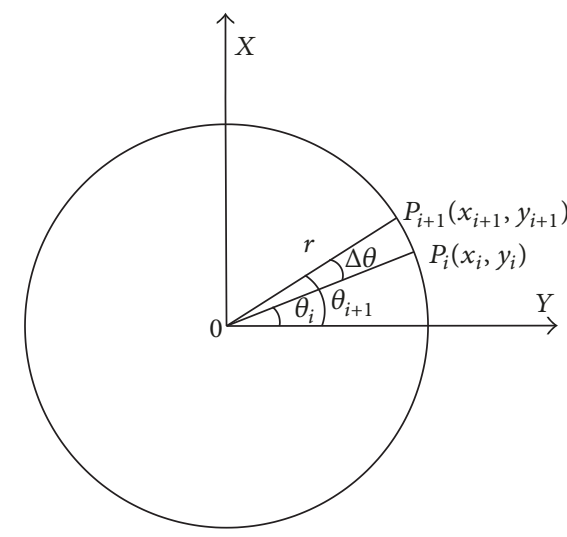

FIgURE 2: The circular interpolation diagram.

3.1. The Circular Interpolation. Let point $P_{i}\left(x_{i}, y_{i}\right)$ and $P_{i+1}\left(x_{i+1}, y_{i+1}\right)$ be the current and next interpolation point on the saddle weld and the angle of the $x$-axis, respectively; $\theta_{i}$ and $\theta_{i+1}$ be the angles between the two points for the $\Delta \theta$, $\theta_{i+1}=\theta_{i}+\Delta \theta$, and the coordinates of the $P_{i}$ point are

$$
\begin{aligned}
& x_{i}=r \sin \theta_{i} \\
& y_{i}=r \cos \theta_{i} .
\end{aligned}
$$

So the coordinates of $P_{i+1}$ can be expressed as

$$
\begin{aligned}
x_{i+1} & =r \sin \left(\theta_{i}+\Delta \theta\right)=r \sin \theta_{i} \cos \Delta \theta-r \cos \theta_{i} \sin \Delta \theta \\
& =y_{i} \cos \Delta \theta-x_{i} \sin \Delta \theta \\
y_{i+1} & =r \cos \left(\theta_{i}+\Delta \theta\right) \\
& =r \cos \theta_{i} \cos \Delta \theta-r \sin \theta_{i} \sin \Delta \theta \\
& =x_{i} \cos \Delta \theta-y_{i} \sin \Delta \theta .
\end{aligned}
$$

When $\theta_{i+1} \leq 2 \pi$, then continue interpolating; when $\theta_{i+1}>$ $2 \pi$, to amend the last step $\Delta \theta$, there will be an interpolation stepping $\Delta \theta^{\prime}$; then, $\Delta \theta^{\prime}=2 \pi-\theta_{i}$.

So the new position of the interpolation point projected in the $x O y$ plane can be expressed as

$$
\begin{aligned}
x & =r \sin \left(\theta_{i}+\Delta \theta\right) \\
y & =r \cos \left(\theta_{i}+\Delta \theta\right) \\
\theta_{i+1} & =\theta_{i}+\Delta \theta,
\end{aligned}
$$

where $\theta_{i}$ is the current angle, $\theta_{i+1}$ is the angle of the next point, and $\Delta \theta$ is the added value of the angle.

Based on the principle of space analytic geometry, we have

$$
\begin{aligned}
& x^{2}+y^{2}-r^{2}=0 \\
& x^{2}+z^{2}-R^{2}=0 .
\end{aligned}
$$

To solve (4),

$$
\begin{aligned}
y^{2}-z^{2} & =r^{2}+R^{2}, \\
z & =\sqrt{R^{2}-r^{2}+y^{2}} .
\end{aligned}
$$




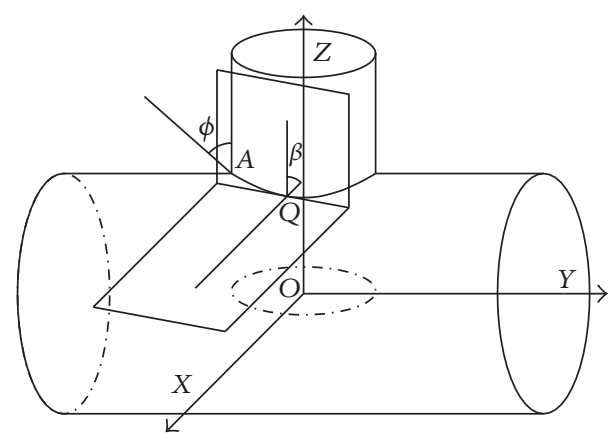

FIGURE 3: Relation diagram between dihedral angle and pose angle.

From (3) and (6), we also have

$$
\begin{aligned}
z & =\sqrt{R^{2}-r^{2}+r^{2} \cos ^{2}\left(\theta_{i}+\Delta \theta\right)} \\
& =\sqrt{R^{2}-r^{2} \sin ^{2}\left(\theta_{i}+\Delta \theta\right)} .
\end{aligned}
$$

3.2. Welding Torch Posture Interpolation. Choose a point in the saddle-shaped weld; dihedral angle of the point on saddle weld is the angle between the cylinder's tangent plane through this point and the pipe's tangent plane. Dihedral angle is not equal at different points on saddle weld. Therefore, $\beta$ is the dihedral angle of point $Q$ on saddle weld (Figure 3). As can be seen from Figure 3, the diagonal angle at the starting point $A$ is $\pi / 2$ and the initial pose angle is $\phi$ (regular angle of welding torch's pose).

Based on the principle of space analytic geometry, the equations of the cylinder and the pipes are given as

$$
\begin{aligned}
& \varphi_{1}(x, y, z)=x^{2}+y^{2}-r^{2}=0 \\
& \varphi_{2}(x, y, z)=x^{2}+z^{2}-R^{2}=0 .
\end{aligned}
$$

Then the normal vectors $\overrightarrow{\mathbf{n}}_{1}$ and $\overrightarrow{\mathbf{n}}_{2}$ at point $Q$ are defined as

$$
\begin{aligned}
& \overrightarrow{\mathbf{n}}_{1}=\left\{\frac{\partial \varphi_{1}}{\partial x}, \frac{\partial \varphi_{1}}{\partial y}, \frac{\partial \varphi_{1}}{\partial z}\right\}=\{x, y, 0\} \\
& \overrightarrow{\mathbf{n}}_{2}=\left\{\frac{\partial \varphi_{2}}{\partial x}, \frac{\partial \varphi_{2}}{\partial y}, \frac{\partial \varphi_{2}}{\partial z}\right\}=\{x, 0, z\}
\end{aligned}
$$

Geometry is defined as

$$
\cos \beta=\frac{\overrightarrow{\mathbf{n}}_{\mathbf{1}} \cdot \overrightarrow{\mathbf{n}}_{\mathbf{2}}}{\left|\overrightarrow{\mathbf{n}}_{\mathbf{1}}\right| \times\left|\overrightarrow{\mathbf{n}}_{\mathbf{2}}\right|}=\frac{x^{2}}{R \times r} .
$$

From (3) and (10), we have

$$
\beta=\arccos \left(\frac{r \sin ^{2}\left(\theta_{i}+\Delta \theta\right)}{R}\right) .
$$

It can be seen from Figure 3 that although dihedral angles of point on saddle weld differ from each other, they all have certain relationship with pose angle. As the rotation axis

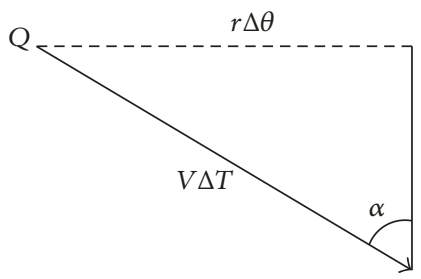

Figure 4: The schematic diagram of linear velocity.

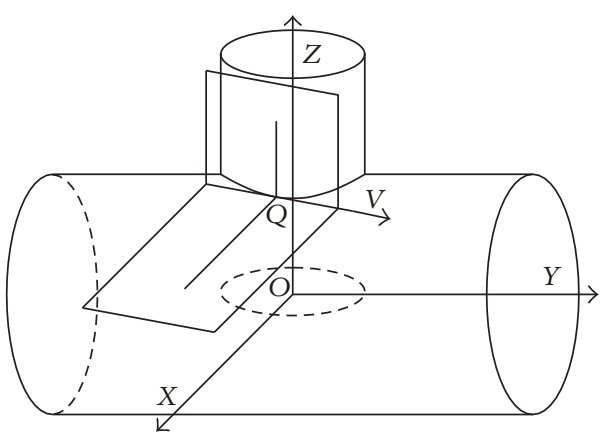

FIGURE 5: Linear velocity direction.

rotates from point $A$ to point $Q$, the dihedral angle changes from the original $\pi / 2$ to $\beta$, and pose angle is supposed to change $\pi / 2-\beta$ in order to meet pose angle requirements. So expression of dihedral angle is as follows: $\delta=\pi / 2-\beta+\phi$. Put formula (11) into it; then

$$
\delta=\frac{\pi}{2}-\arccos \left(\frac{r \sin ^{2}\left(\theta_{i}+\Delta \theta\right)}{R}\right)+\phi .
$$

After the rotation angle changes $\Delta \theta$ from $\theta_{i}$ to $\theta_{i+1}$, according to (3), (7) and (12) can calculate the coordinates of the new interpolation point $P_{i+1}\left(x, y, z, \theta_{i+1}, \delta\right)$. Selecting the appropriate $\Delta \theta$, one can obtain interpolation saddle weld by providing the cylinder and the pipe radius $R, r$, welding torch starting pose angle $\phi$.

3.3. Control Law of Variable Angle $\Delta \theta$. The control of the interpolation stepping angle $\Delta \theta$ is divided into equal angle interpolation and variable angle interpolation. Equal angle interpolation mode is to keep the stepping angle $\Delta \theta$ constant, and actual welding linear velocity of welding torch is not constant, affecting the quality of welding. Variable angle interpolation is to make every step interpolation stepping angle $\Delta \theta$ with $\theta$ to make a change. In the $\Delta T$ fixed conditions, welding linear velocity is constant and each step of the interpolation stepping-length will be equal, to ensure the welding of high quality.

Select a point $Q$ on the saddle-shaped weld; according to kinematics principle, linear velocity direction of the point $Q$ is direction vector of intersection line of two intersection's tangent planes at point $Q$ (Figure 5). Suppose linear velocity of welding torch is $V$, interpolation cycle is $\Delta T$, and the angle between the $z$-axis and linear velocity is $\alpha$ (shown in Figure 4). 
As can be seen from Figure 4,

$$
\begin{aligned}
r \cdot \Delta \theta & =v \cdot \Delta T \cdot \sin \alpha \\
\Delta \theta & =\frac{v \cdot \Delta T \cdot \sin \alpha}{r} .
\end{aligned}
$$

Set the coordinates of the $Q$ point $Q\left(x_{q}, y_{q}, z_{q}\right)$.

The tangent plane equation of the cylinder at the point $Q$ is expressed as

$$
x_{q} \cdot\left(x-x_{q}\right)+z_{q} \cdot\left(z-z_{q}\right)=0 \text {. }
$$

And then

$$
x_{q} \cdot x+z_{q} \cdot z=x_{q}^{2}+z_{q}^{2}=R^{2} .
$$

Similarly the tangent plane equation of the pipe at the point $Q$ is expressed as

$$
x_{q} \cdot x+y_{q} \cdot y=x_{q}^{2}+y_{q}^{2}=r^{2} .
$$

Equations of intersection line of two tangent plans are

$$
\begin{aligned}
& x_{q} \cdot x+z_{q} \cdot z=x_{q}^{2}+z_{q}^{2}=R^{2} \\
& x_{q} \cdot x+y_{q} \cdot y=x_{q}^{2}+y_{q}^{2}=r^{2} .
\end{aligned}
$$

The two points $Q\left(x_{q}, y_{q}, z_{q}\right)$ and $\left(0, r^{2} / y_{q}, R^{2} / z_{q}\right)$ are on the intersection line of two tangent planes, and the direction vector of the tangent line equation is calculated as

$$
\overrightarrow{\mathbf{n}}_{3}=\left(-x_{q}, \frac{r^{2}}{y_{q}}-y_{q}, \frac{R^{2}}{z_{q}}-z_{q}\right)=\left(-x_{q}, \frac{x_{q}{ }^{2}}{y_{q}}, \frac{x_{q}{ }^{2}}{z_{q}}\right) .
$$

The direction vector of the $z$-axis is $\overrightarrow{\mathbf{n}}_{\mathbf{4}}=(0,0,1)$; one can get following equation:

$$
\cos \alpha=\frac{\overrightarrow{\mathbf{n}}_{3} \cdot \overrightarrow{\mathbf{n}}_{4}}{\left|\overrightarrow{\mathbf{n}}_{3}\right|\left|\overrightarrow{\mathbf{n}}_{4}\right|}=\frac{x_{q} / z_{q}}{\sqrt{1+\left(x_{q} / y_{q}\right)^{2}+\left(x_{q} / z_{q}\right)^{2}}}
$$

Then

$$
\sin \alpha=\sqrt{1-\cos ^{2} \alpha}=\frac{\sqrt{1+\left(x_{q} / y_{q}\right)^{2}}}{\sqrt{1+\left(x_{q} / y_{q}\right)^{2}+\left(x_{q} / z_{q}\right)^{2}}} .
$$

And because of the $Q$ point in the saddle-shaped space curve, according to (3), we have

$$
\begin{aligned}
& \frac{x_{q}}{y_{q}}=\tan \left(\theta_{i}+\Delta \theta\right) \\
& \frac{x_{q}}{z_{q}}=\frac{r \sin \left(\theta_{i}+\Delta \theta\right)}{\sqrt{R^{2}-r^{2} \sin ^{2}\left(\theta_{i}+\Delta \theta\right)}} .
\end{aligned}
$$

Substituting (22) into (21),

$$
\sin \alpha=\frac{\sqrt{1+\tan ^{2}\left(\theta_{i}+\Delta \theta\right)}}{\sqrt{\left(r \sin \left(\theta_{i}+\Delta \theta\right)\right)^{2} /\left(R^{2}-\left(r \sin \left(\theta_{i}+\Delta \theta\right)\right)^{2}\right)+\tan ^{2}\left(\theta_{i}+\Delta \theta\right)+1}} .
$$

Substituting (23) into (14),

$$
\Delta \theta=\frac{v \cdot \Delta T \cdot \sqrt{1+\tan ^{2}\left(\theta_{i}+\Delta \theta\right)}}{r \cdot \sqrt{\left(r \sin \left(\theta_{i}+\Delta \theta\right)\right)^{2} /\left(R^{2}-\left(r \sin \left(\theta_{i}+\Delta \theta\right)\right)^{2}\right)+\tan ^{2}\left(\theta_{i}+\Delta \theta\right)+1}} .
$$

From (24), interpolation step angle $\Delta \theta$ changes in real time with the change of $\theta_{i}$ to ensure that practical linear velocity of the welding torch is kept constant so as to meet the requirement of the welding process.

\section{Simulation Results}

In order to verify the correctness of the mathematical model and algorithm, using programming takes simulation experiment, taking orthogonal saddle welding, for example, assume that practical welding parameters are as follows: the radius of cylinder $R=600 \mathrm{~mm}$, the radius of pipe $r=$ $150 \mathrm{~mm}$, the starting pose angle $=\pi / 4$, and $\Delta T=0.004 \mathrm{~s}$. MATLAB simulation results are shown in Figures 6-11. The interpolation data of $\theta$ and $(x, y, z, \delta)$ by algorithm are shown in Table 1.

\section{Welding Experiment}

According to the welding process of the saddle weld, the main parameters of the welding experiment are listed in 
TABLE 1: The interpolation data.

Interpolation Rotation angle $\theta / \mathrm{rad}$ Coordinate of $x \quad x / \mathrm{mm}$ Coordinate of $y \quad y / \mathrm{mm}$ Coordinate of $z z / \mathrm{mm} \quad$ Pose angle of torch $\delta / \mathrm{mm}$ times

\begin{tabular}{|c|c|c|c|c|c|}
\hline (1) & 0.0000 & 0.0000 & 150.0000 & 600.0000 & 0.7854 \\
\hline (2) & 0.1571 & 23.4652 & 148.1533 & 599.5410 & 0.7915 \\
\hline (3) & 0.3142 & 46.3525 & 142.6585 & 598.2069 & 0.8093 \\
\hline (4) & 0.4712 & 68.0986 & 133.6510 & 596.1230 & 0.8369 \\
\hline (5) & 0.6283 & 88.1678 & 121.3525 & 593.4867 & 0.8719 \\
\hline (6) & 0.7854 & 106.0660 & 106.0660 & 590.5506 & 0.9107 \\
\hline (7) & 0.9425 & 121.3525 & 88.1678 & 587.5998 & 0.9498 \\
\hline (8) & 1.0996 & 133.6510 & 68.0986 & 584.9251 & 0.9852 \\
\hline (9) & 1.2566 & 142.6585 & 46.3525 & 582.7938 & 1.0135 \\
\hline (10) & 1.4137 & 148.1533 & 23.4652 & 581.4212 & 1.0318 \\
\hline (11) & 1.5708 & 150.0000 & 0.0000 & 580.9475 & 1.0381 \\
\hline (12) & 1.7279 & 148.1533 & -23.4652 & 581.4212 & 1.0318 \\
\hline (13) & 1.8850 & 142.6585 & -46.3525 & 582.7938 & 1.0135 \\
\hline (14) & 2.0420 & 133.6510 & -68.0986 & 584.9251 & 0.9852 \\
\hline (15) & 2.1991 & 121.3525 & -88.1678 & 587.5998 & 0.9498 \\
\hline (16) & 2.3562 & 106.0660 & -106.0660 & 590.5506 & 0.9107 \\
\hline (17) & 2.5133 & 88.1678 & -121.3525 & 593.4867 & 0.8719 \\
\hline (18) & 2.6704 & 68.0986 & -133.6510 & 596.1230 & 0.8369 \\
\hline (19) & 2.8274 & 46.3525 & -142.6585 & 598.2069 & 0.8093 \\
\hline (20) & 2.9845 & 23.4652 & -148.1533 & 599.5410 & 0.7915 \\
\hline$(21)$ & 3.1416 & 0.0000 & -150.0000 & 600.0000 & 0.7854 \\
\hline$(22)$ & 3.2987 & -23.4652 & -148.1533 & 599.5410 & 0.7915 \\
\hline (23) & 3.4558 & -46.3525 & -142.6585 & 598.2069 & 0.8093 \\
\hline (24) & 3.6128 & -68.0986 & -133.6510 & 596.1230 & 0.8369 \\
\hline (25) & 3.7699 & -88.1678 & -121.3525 & 593.4867 & 0.8719 \\
\hline (26) & 3.9270 & -106.0660 & -106.0660 & 590.5506 & 0.9107 \\
\hline (27) & 4.0841 & -121.3525 & -88.1678 & 587.5998 & 0.9498 \\
\hline (28) & 4.2412 & -133.6510 & -68.0986 & 584.9251 & 0.9852 \\
\hline (29) & 4.3982 & -142.6585 & -46.3525 & 582.7938 & 1.0135 \\
\hline (30) & 4.5553 & -148.1533 & -23.4652 & 581.4212 & 1.0318 \\
\hline$(31)$ & 4.7124 & -150.0000 & 0.0000 & 580.9475 & 1.0381 \\
\hline$(32)$ & 4.8695 & -148.1533 & 23.4652 & 581.4212 & 1.0318 \\
\hline (33) & 5.0265 & -142.6585 & 46.3525 & 582.7938 & 1.0135 \\
\hline (34) & 5.1836 & -133.6510 & 68.0986 & 584.9251 & 0.9852 \\
\hline (35) & 5.3407 & -121.3525 & 88.1678 & 587.5998 & 0.9498 \\
\hline (36) & 5.4978 & -106.0660 & 106.0660 & 590.5506 & 0.9107 \\
\hline (37) & 5.6549 & -88.1678 & 121.3525 & 593.4867 & 0.8719 \\
\hline$(38)$ & 5.8119 & -68.0986 & 133.6510 & 596.1230 & 0.8369 \\
\hline (39) & 5.9690 & -46.3525 & 142.6585 & 598.2069 & 0.8093 \\
\hline (40) & 6.1261 & -23.4652 & 148.1533 & 599.5410 & 0.7915 \\
\hline (41) & 6.2832 & 0.0000 & 150.0000 & 600.0000 & 0.7854 \\
\hline
\end{tabular}

Table 2. The photograph of the saddle seam surface is shown in Figures 12 and 13.

Correct saddle seam is reflected in the photos, which verify the feasibility of the interpolation algorithm.

\section{Conclusions}

According to the welding technology of saddle seam, the working principle of auto-welding machine for saddle-shaped 


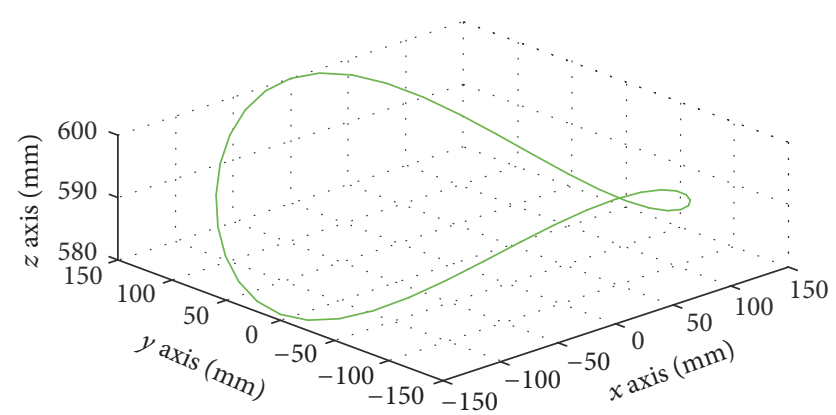

FIGURE 6: Simulation result of the trajectory interpolation algorithm.

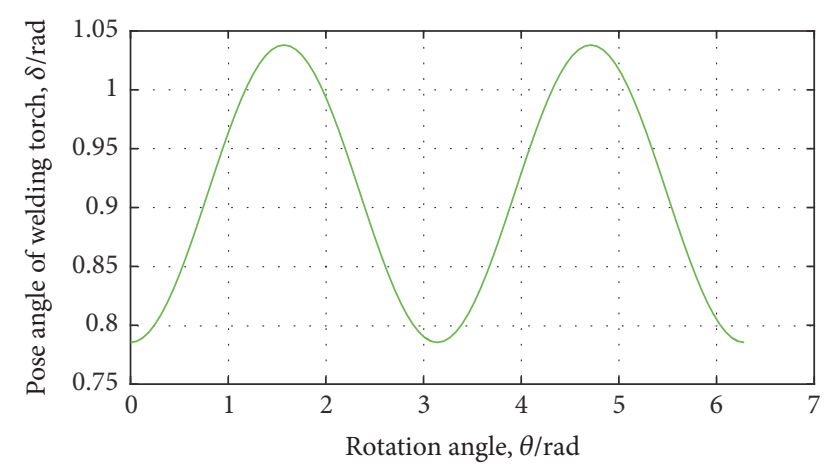

FIGURE 7: Simulation figure of the relation between rotation angle and pose angle.

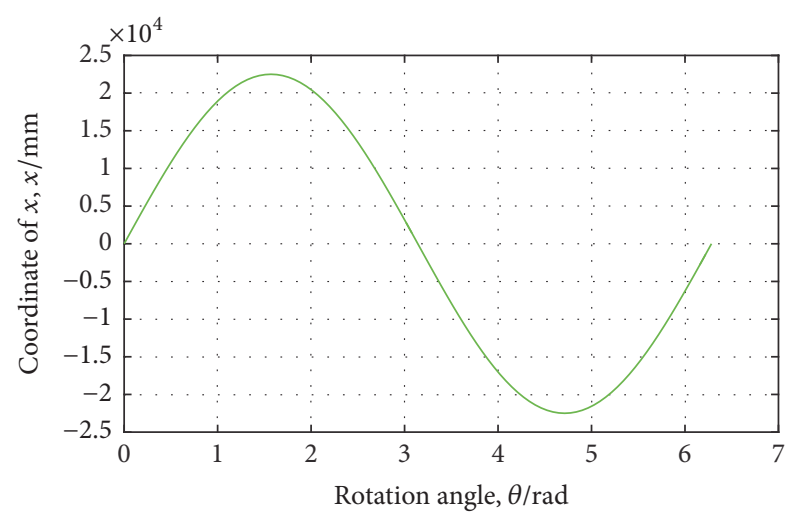

FIGURE 8: Simulation figure of the relationship between rotation angle and $x$-axis coordinate value.

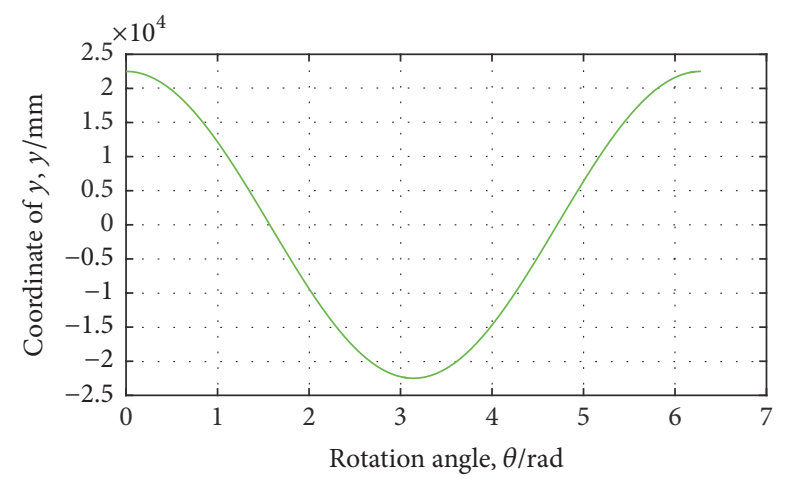

FIGURE 9: Simulation figure of the relationship between rotation angle and $y$-axis coordinate value.

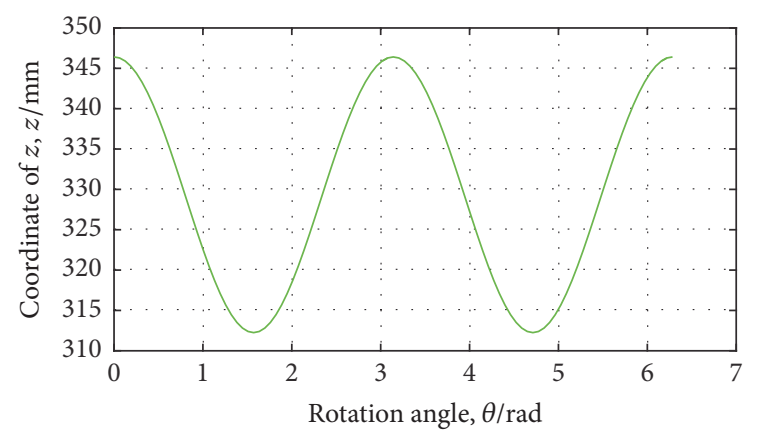

FIGURE 10: Simulation figure of the relationship between rotation angle and $z$-axis coordinate value.

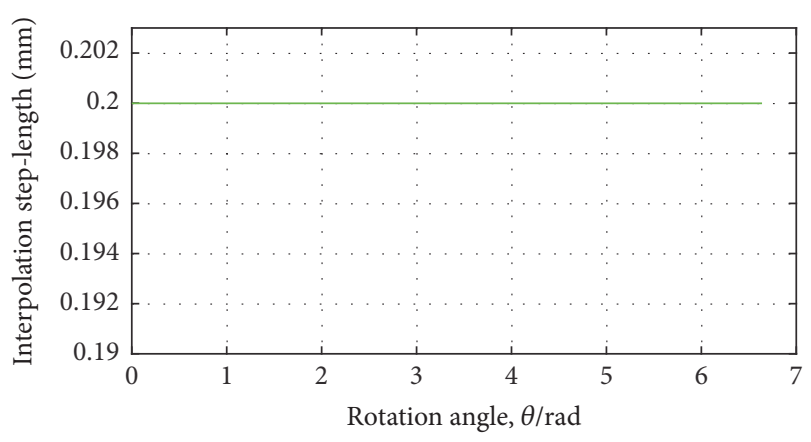

FIGURE 11: Relationship between rotation angle and interpolation step-length.

TABLE 2: Welding experiment parameters.

\begin{tabular}{lc}
\hline Parameter & Value \\
\hline Radius of cylinder & $600 \mathrm{~mm}$ \\
Radius of branch pipe & $150 \mathrm{~mm}$ \\
Welding type & GMAW \\
Welding current & $230 \mathrm{~A}$ \\
Arc voltage & $25.3 \mathrm{~V}$ \\
Welding speed & $80 \mathrm{~mm} / \mathrm{min}$ \\
Wire feed rate & $6 \mathrm{~m} / \mathrm{min}$ \\
Wire diameter & $1.2 \mathrm{~mm}$ \\
Pose angle & $25^{\circ}$ \\
Wire extension & $12 \mathrm{~mm}$ \\
\hline
\end{tabular}

weld is proposed, and the mathematical model and interpolation algorithm of saddle-shaped weld are established.

The interpolation algorithm can well meet the technical requirement of automatic welding and can achieve trajectory control of welding of saddle-shaped weld and can also achieve control of welding torch's pose, to ensure better welding conditions.

A variable angle interpolation method is used for realtime change interpolation stepping angle and to guarantee that practical linear velocity of the welding torch is kept constant and improve the quality of the weld seam. 


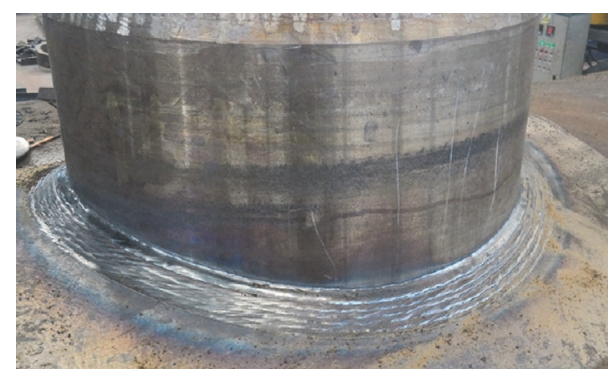

Figure 12: Photograph of the saddle seam surface.

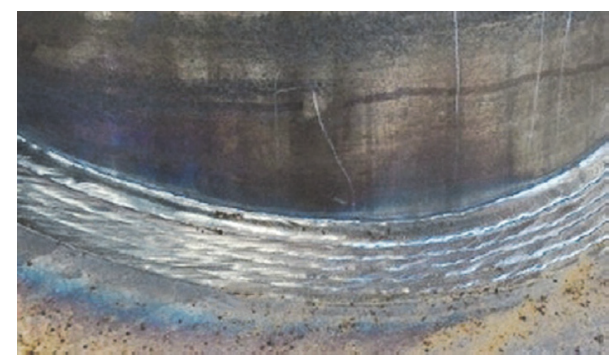

Figure 13: Photograph of the saddle seam surface.

\section{Conflicts of Interest}

The authors declare that there are no conflicts of interest regarding the publication of this paper.

\section{Acknowledgments}

This research was supported by Xinjiang Production and Construction Corps Science and Technology Research and Achievement Transformation Project under Grant no. 2016AB004.

\section{References}

[1] D. Tiequn, M. Fanrong, and S. Guanyuan, "Kinematic simulation on large cylinder saddle automatic welding machine base on ADAMS," in Welding \& Joining, pp. 64-66, 2008.

[2] Hong Feng Wang, A Spreading Algorithm of an Intersecting Line.Computer Development \& Applications, PP. 59-62, 2009.

[3] X. Tian, X. Yi, and H. Chang, "Interpolation of saddle-shaped trajectory in non-Cartesian coordinate system," Advanced Science Letters, vol. 19, no. 7, pp. 1927-1930, 2013.

[4] M.-Y. Huo, X.-G. Wang, and P. Yin, "Real-time interpolation algorithm and simulation of seam of intersection line for automatic welding," Hanjie Xuebao/Transactions of the China Welding Institution, vol. 27, no. 11, pp. 37-40, 2006.

[5] F. Ren, S. Chen, S. Yin, and X. Guan, "Modeling on weld position and welding torch pose in welding of intersected pipes," Hanjie Xuebao/Transactions of the China Welding Institution, vol. 29, no. 11, pp. 33-36, 2008.

[6] X. Zhiming, C. Jincheng, and F. Zhengjin, "Performance evaluation of a real-time interpolation algorithm for NURBS curves," The International Journal of Advanced Manufacturing Technology, vol. 20, no. 4, pp. 270-276, 2002.
[7] W. T. Lei, M. P. Sung, L. Y. Lin, and J. J. Huang, "Fast realtime NURBS path interpolation for CNC machine tools," International Journal of Machine Tools \& Manufacture, vol. 47, no. 10, pp. 1530-1541, 2007.

[8] H. Dong, B. Chen, Y. Chen, J. Xie, and Z. Zhou, "An accurate NURBS curve interpolation algorithm with short spline interpolation capacity," The International Journal of Advanced Manufacturing Technology, vol. 63, no. 9-12, pp. 1257-1270, 2012.

[9] S.-I. Gofuku, S. Tamura, and T. Maekawa, "Point-tangent/pointnormal B-spline curve interpolation by geometric algorithms," Computer-Aided Design, vol. 41, no. 6, pp. 412-422, 2009.

[10] H.-Y. Tam, H. Xu, and P. W. Tse, "An algorithm for the interpolation of hybrid curves," Computer-Aided Design, vol. 35, no. 3, pp. 267-277, 2003.

[11] G. Srijuntongsiri, "An iterative/subdivision hybrid algorithm for curve/curve intersection," The Visual Computer, vol. 27, no. 5, pp. 365-371, 2011.

[12] H. Qi, X. Tian, X. Zhang, and B. Peng, "Interpolation algorithm for saddle-shaped curve auto-welding based on angle approaching," Hanjie Xuebao/Transactions of the China Welding Institution, vol. 28, no. 3, pp. 93-96, 2007.

[13] L. Yan, T. Xincheng, X. Qing, and P. Bo. Four-axis interpolation, "algorithm for saddle-shaped curve automated welding," in P.Bo. Four-axis interpolation algorithm for saddle-shaped curve automated welding.Transactions of the China Welding Institution, vol. 30, pp. 81-84, Transactions of the China Welding Institution, 2009.

[14] D. Weimin, G. Gmmbin, and S. Dongming, "Study on the errors and real-time of interpolation methods," Machine Building \& Automation, pp. 18-21, 2006.

[15] Y. Changlong, L. Lix, and Z. Yonghui, "Simulation of welding intersecting cylinders line for welding robot," Journal of Shen Yang University of Technology, vol. Vol, no. 05, pp. 426-429, 2003. 


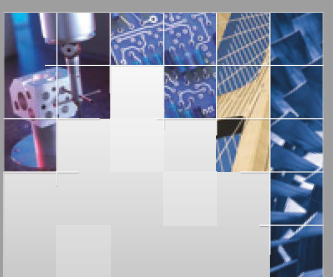

\section{Enfincering}
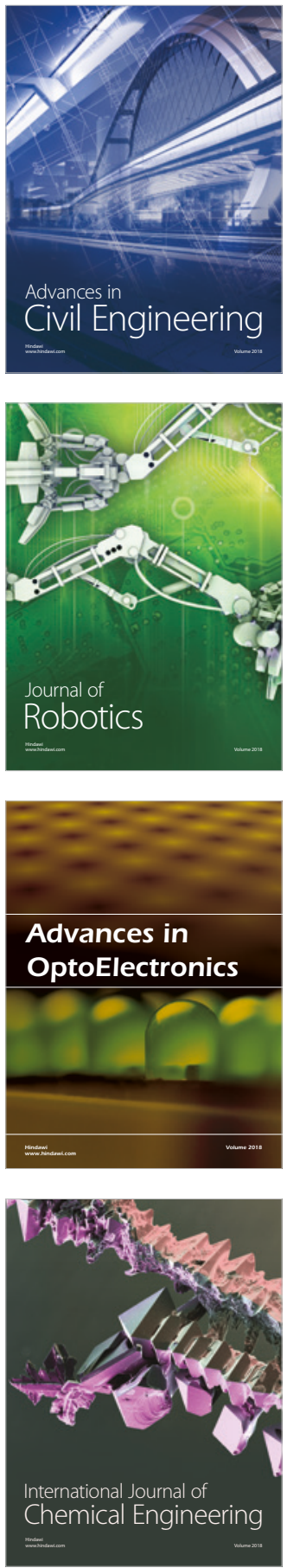

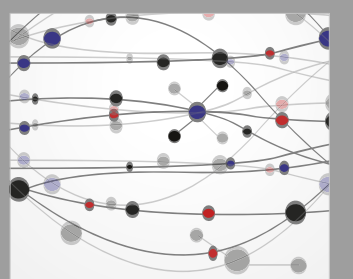

\section{Rotating \\ Machinery}

The Scientific World Journal

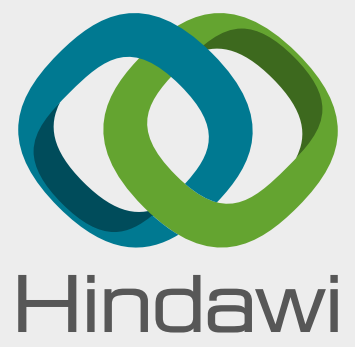

Submit your manuscripts at

www.hindawi.com
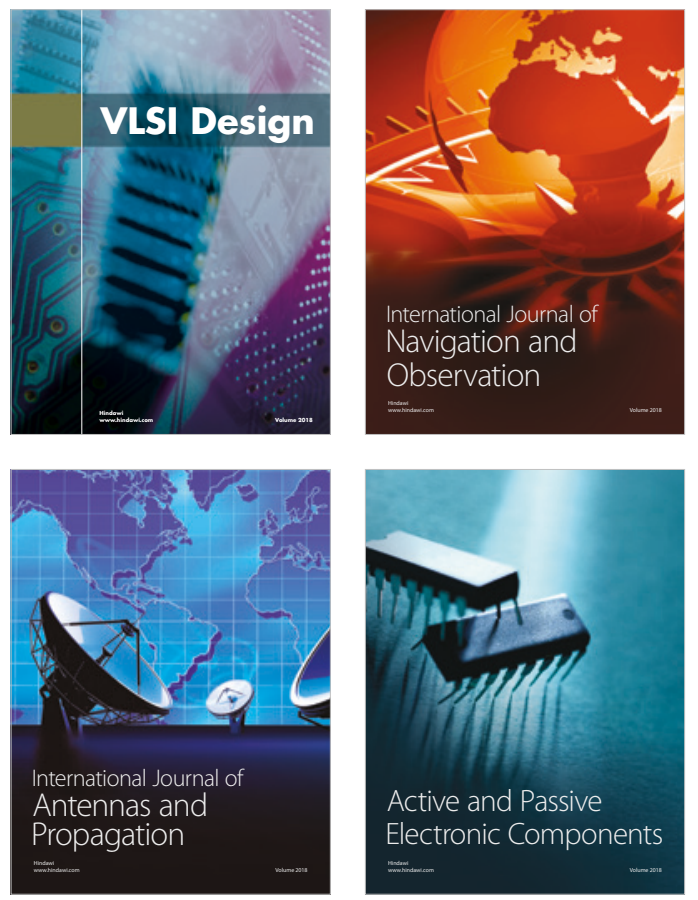
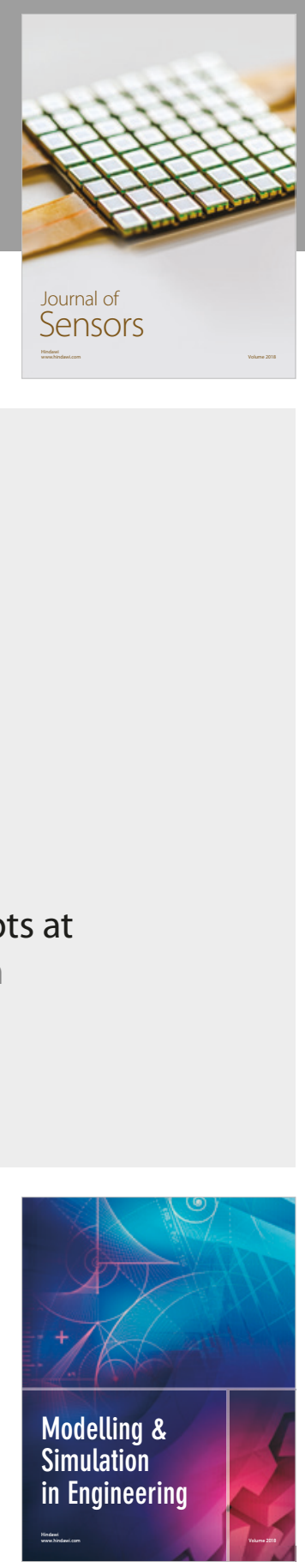

\section{Advances \\ Multimedia}
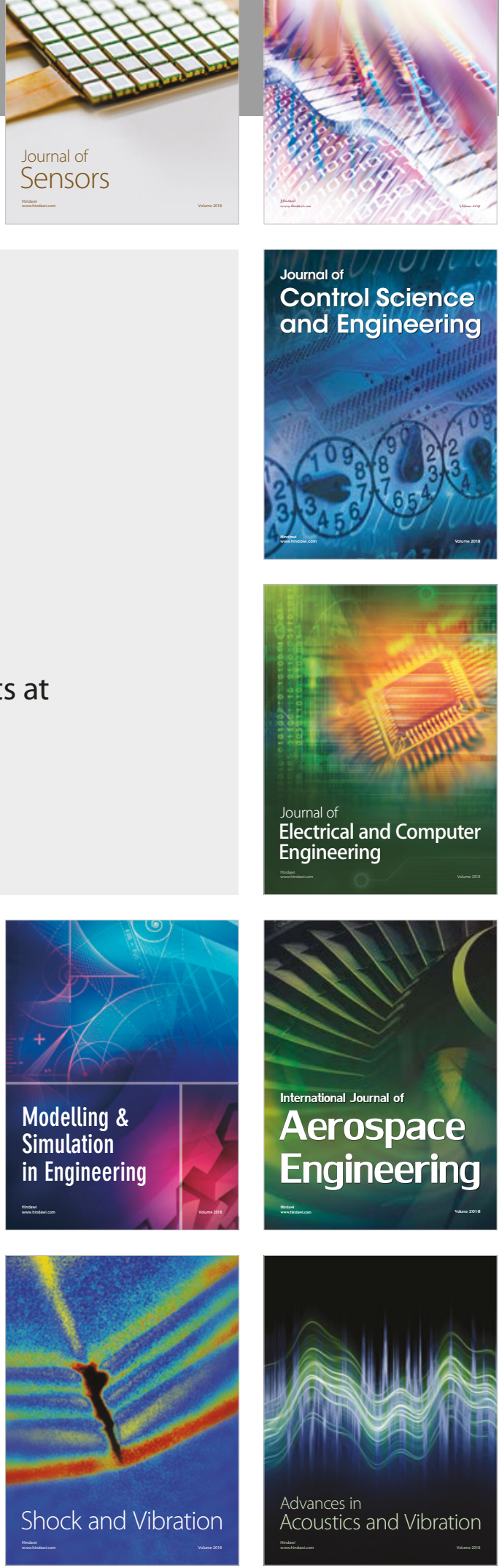